\title{
Implementation of a Generic Type I Wind Turbine Generator for Power System Stability Studies
}

\author{
R. Villena-Ruiz ${ }^{1}$, A. Lorenzo-Bonache ${ }^{1}$, A. Honrubia-Escribano ${ }^{1}$, E. Gómez-Lázaro ${ }^{1}$ \\ ${ }^{1}$ Department of Wind Energy and Power Systems \\ Renewable Energy Research Institute and DIEEAC/EDII-AB, Universidad de Castilla-La Mancha, \\ 02071 Albacete, Spain \\ Phone/Fax number: +0034 967599200; ext=96259, e-mail: $\underline{\text { Raquel.Villena@alu.uclm.es }}$
}

\begin{abstract}
According to the International Electrotechnical Commission (IEC), the standardization and modelling of generic Wind Turbine Generators (WTG) is a key fact which allows the co-operation of international organizations in the electrical field.
\end{abstract}

Thus, according to the IEC 61400-27-1, the generic Type 1 WTG model has been implemented and simulated in DIgSILENTPowerFactory (PF). In fact, PF is the preferred software tool for some of the European network operators. The process of structuring and construction of the different WTG components within PF are conveniently described in this paper.

In order to correctly simulate the generic IEC WTG model in PF, aerodynamic and mechanical systems are also required. For this reason, the Dynamic Simulation Language (DSL) tool within PF has been used for modelling these non-electrical components. Moreover, this language allows the dynamic modelling of linear and non-linear systems.

Finally, once all the WTG components have been defined, normal and fault conditions have been analyzed in order to study the WTG dynamic response.

\section{Key words}

DIgSILENT-PowerFactory, Dynamic Simulation Language, International Electrotechnical Commission, Standard Model, Wind Turbine Generator.

\section{Introduction}

It is clear that in an increasingly polluted world due to the use of fossil fuels, wind power plays a very important role. Among the many advantages of wind power, the fact that it is a renewable and sustainable energy source is one of the most important one.

Therefore, the continuous process of invention, renovation and improvement is what makes this kind of power source a very interesting field.

However, the existing issues when interconnecting wind farms to the electrical network (such as power fluctuations), cause serious stability problems. With the objective of improving the system stability, the dynamic simulation of power system events has been performed. In order to study the behaviour of these IEC-designed WTGs, normal operation and fault condition situations have been analyzed. As previously mentioned, PF has been the used simulation tool. It is one of the most powerful programs in power system modelling, being clearly one of the leaders in the Electrical Engineering field.

Finally, it has to be noted that the obtained results clearly show that generic models behave similarly to actual WTGs [1]-[4]. In fact, due to the recent publication of the IEC 61400-27-1 (Feb 2015), these kind of analysis are really important, trying to get a wide use of the generic WTG models [5].

\section{Model description}

It must be considered that the IEC standard WTG models try to combine the main characteristics of the existing WTG types, also covering the possibility of developing future WTG models through these generic types.

In the present work, a type 1 WTG is going to be briefly described. It consists of an asynchronous generator with fixed rotor resistance (what is usually known as squirrel cage), which is directly connected to the grid. It can be divided into two models, depending on its blade pitch angles (fixed or controllable) [6]. The main blocks that are part of the WTG are:

A. Aerodynamic

It is considered a constant value of the torque for Type 1A WTG. For Type IB WTG the aerodynamic effects are embedded in the Blade Angle Control Model.

B. Mechanical

The mechanical block is usually implemented as a two-mass model. It can be distinguished two parts within this two-mass model: the low speed 
side (comprising the WTG rotor), and the high speed side (comprising the generator shaft).

\section{Generator system}

Based on IEC 61400-27-1 guidelines, it has been used one of the standard induction generators available in $P F$.

\section{DIgSILENT - PowerFactory. Dynamic Simulation Language (DSL)}

For a proper modelling of the different WTG blocks using DSL, it is highly recommended to follow these steps:

- Data collection. All the known electrical and nonelectrical variables of the block have to be clearly distinguished and defined.

- Modelling. In order to correctly define the block model, all the data collection previously made has to be used for defining the blocks diagrams. They are formed in turn by differential equations or transfer functions. There are two ways of developing models with PF: through DSL, or by using predefined macros.

- Initialization process. If necessary, the user has to identify the initial conditions of the variables, verifying also the syntax routines [7][8].

It is important to note some important aspects relating to some of the above steps, such as the calculation of the initial conditions [7]. The correct initialization of the WTG model will avoid fictitious transients, evaluating correctly the dynamic response of the system. Thus, the first step that has to be done is to perform a load flow calculation. It will provide the steady state condition of the simulated power system. Besides, all the electrical components will be initialized in this way. After that, the initialization process of the non-electrical components begins. For this purpose, some mathematical calculations have to be carried out: setting to zero the derivatives of the state variables; and defining the unknown input and output signals in terms of the known variables (usually stablished from the load flow calculation).

Now, the graphical modelling of each WTG block has been performed, following the next steps [9][10]:

- Define the location of the new model within PF

- $\quad$ Generate a new Block/Frame Diagram.

- Draw the blocks and the operators.

- Assign built-in and/or user defined macros to the blocks.

- Connect all the signals.

- Calculate each model's initial conditions (previously explained).
- Set the model's initial conditions in the main block.

\section{- $\quad$ Generate the Common Model.}

Once all the system's blocks have been defined, a Composite Frame has been created. It relates each model (aerodynamic, mechanical...) with the asynchronous generator. Finally, the Composite Frame created will be related to a new Composite Model.

To better understand the DIgSILENT Simulation Language, it is recommended to read the PowerFactory DSL tutorial [11], although a schematic representation of the hierarchical system just described is shown in Figure 1:

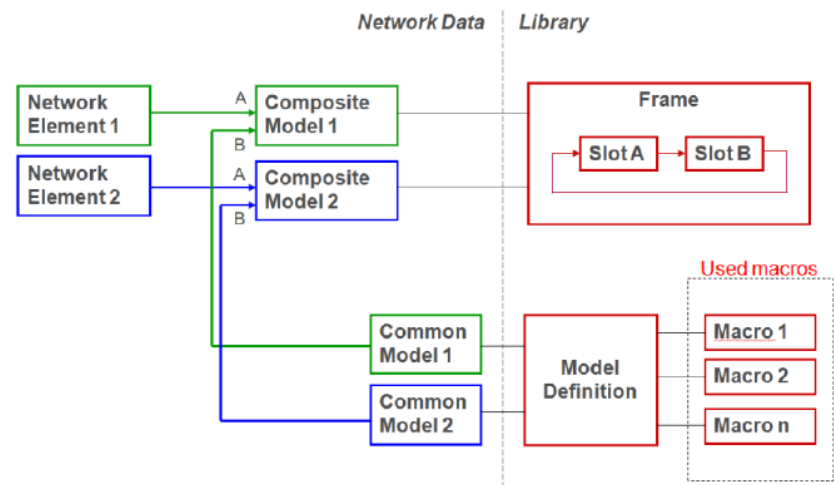

Figure 1. Hierarchical System of DIgSILENT Simulation Language Source: PowerFactory-DSL Tutorial [11]

\subsection{Frame Diagrams}

Following the steps described in the previous section, the Frame Diagrams for both types of WTG have been built. Regarding this point, special attention must be paid to the setting of the equations that define the variables' values and names, and also to those that define the initial conditions of the input, output and state variables signals.

Once all these initial conditions have been set and checked, the model is ready for being used as a part of the Composite Frame that describes the behaviour of the whole WTG. In the next figures, the Frame Diagrams that are part of the Types 1A and 1B WTG are shown:

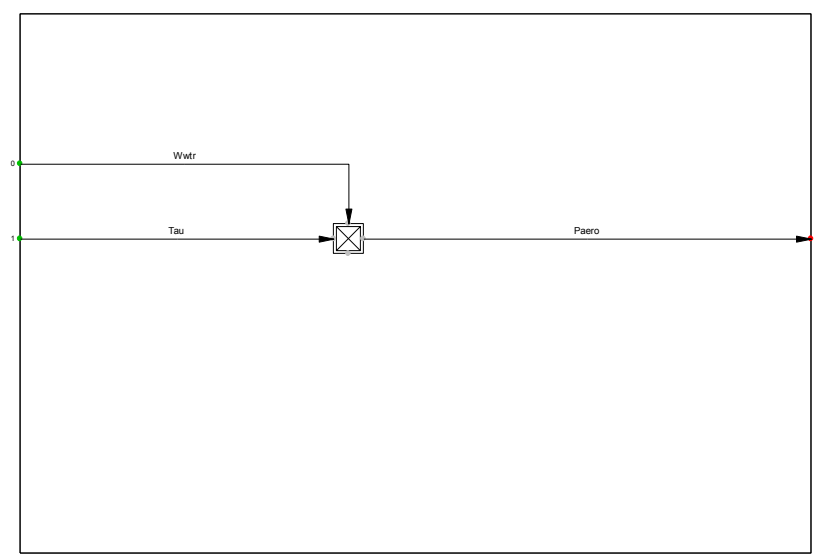

Figure 2. Aerodynamic Frame Diagram for Type 1A WTG 
It is important to note that it has been used a built-in generator model from PF, which already considers its own inertia. As the original two-mass mechanical model considers this inertia value as part of the control system, it has been necessary to make some changes to it [6]. Thus, the exchanged signals between the two-mass model and the generator model have varied with respect to the original design from the IEC Standard. Nevertheless, this is something that does not really matter, because the final results are exactly the same. This new mechanical Frame Diagram is the same for both Type 1A and Type 1B WTG, as it can be seen in Figure 3.

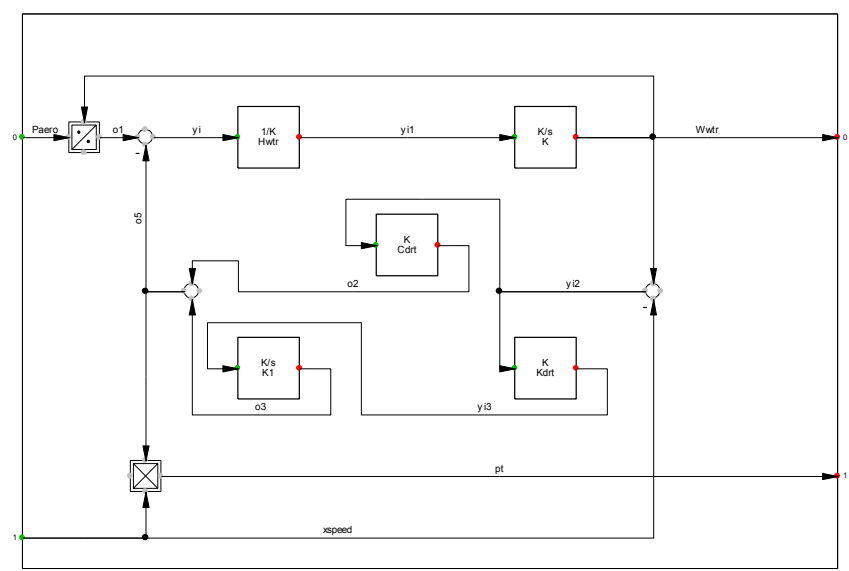

Figure 3. Mechanical Frame Diagram for Type 1A and Type 1B WTG

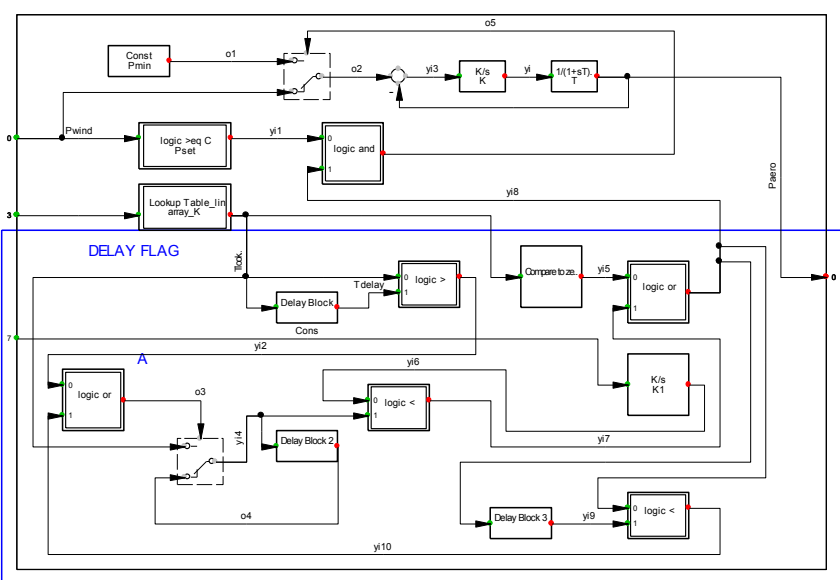

Figure 4. Blade Angle Control Frame Diagram for Type 1B WTG

\subsection{Composite Frames}

Once all the above steps have been followed and all the Frame Diagrams have been created, it has been possible to build the Composite Frames for types 1A and 1B WTG, which define all the existing relationships between the signals of each model, as it is shown in Figure 5.

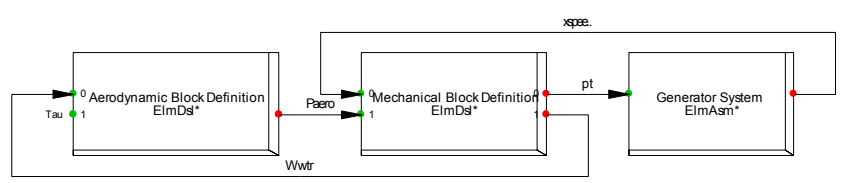

Figure 5. Type 1A WTG Composite Frame

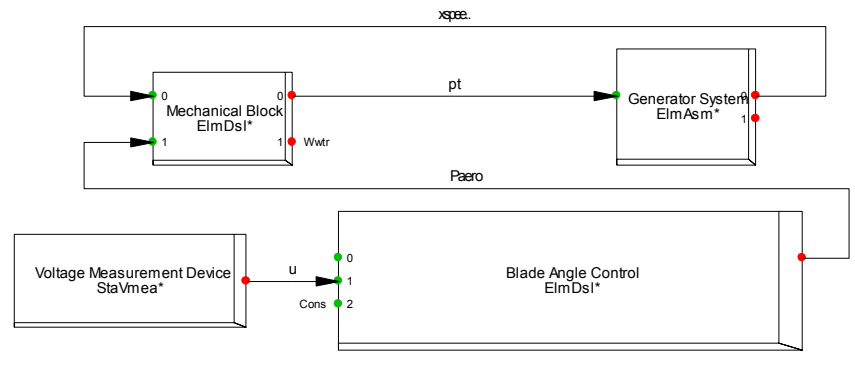

Figure 6. Type 1B WTG Composite Frame

As it has been mentioned before, each Frame Diagram has been associated to its corresponding Slot in the Composite Frame. There is no a Frame Diagram for the Generator System because these Slots are filled with the corresponding Standard Induction Generators, placed on the network grids used in PF. The simplified power system used for testing both models is shown in Figure 7.

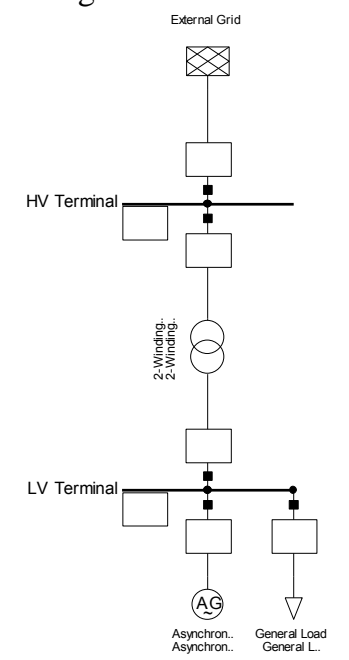

Figure 7. Single line diagram of the study cases

\section{Results and discussion}

Now, the behaviour of both WTG types has to be studied. Thus, the simulation method used for this purpose is the "Balanced RMS Simulation". This RMS (Root Mean Square) simulation function is mainly used for stability analysis, and it considers dynamics in the electromechanical and control devices. Furthermore, a symmetrical and a steady-state representation of the passive electrical network is used. The time that PF requires for executing the simulation when using the RMS function is lower than the one required when using the EMT (Electro-Magnetic Transient) simulation function, mainly due to the RMS method only takes into account the fundamental components of voltages and currents [12], and due to the step size has a lower value in the second case.

Both models have been tested for normal and fault conditions. As there is no possibility of changing the blade angle in Type 1A, the only way of modifying the active power is varying the aerodynamic torque [6]. This allows observing the transient periods of the active and reactive power. Otherwise, the transients are clearly visible in Type 1B WTG when initializing and simulating the model. 


\subsection{Normal Condition}

The aerodynamic torque has been set to 0.9 p.u. at $10 \mathrm{~s}$, and then set to 1.18 p.u. at $25 \mathrm{~s}$ during the simulation in Type 1A. The system response regarding the active and reactive powers is shown in Figures 8 and 9.

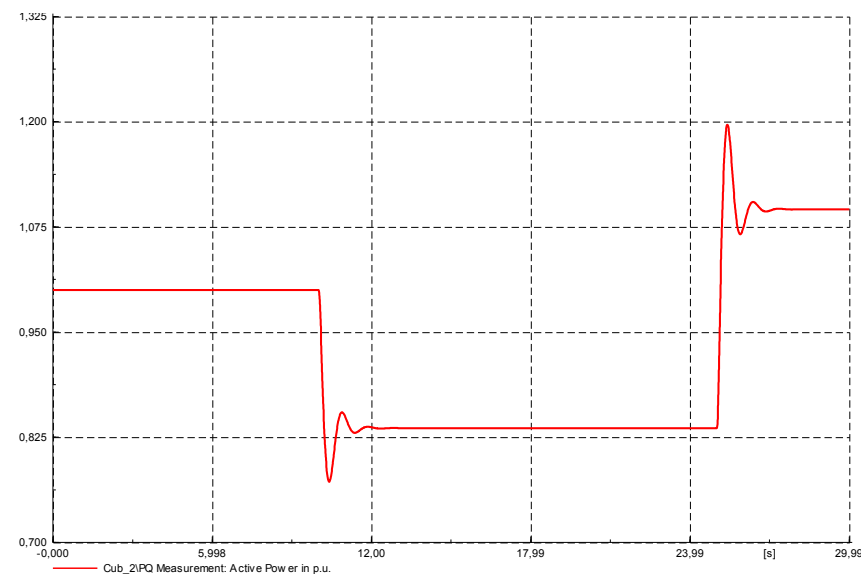

Figure 8. Active Power during normal condition for Type 1A WTG

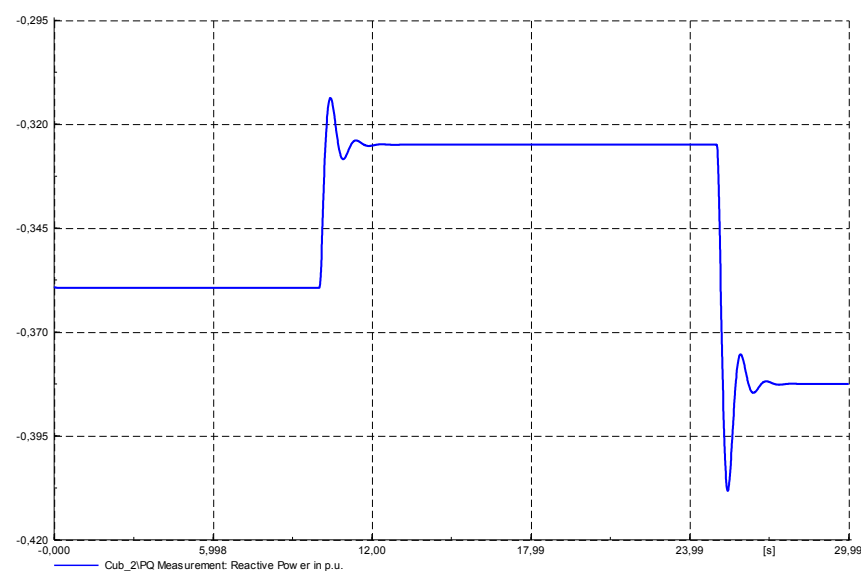

Figure 9. Reactive Power during normal condition for Type 1A WTG

The simulation during normal conditions of Type 1B leads the model to have some transient periods initially, until all the variables have been correctly adjusted. Figures 10 and 11 show those transient periods.

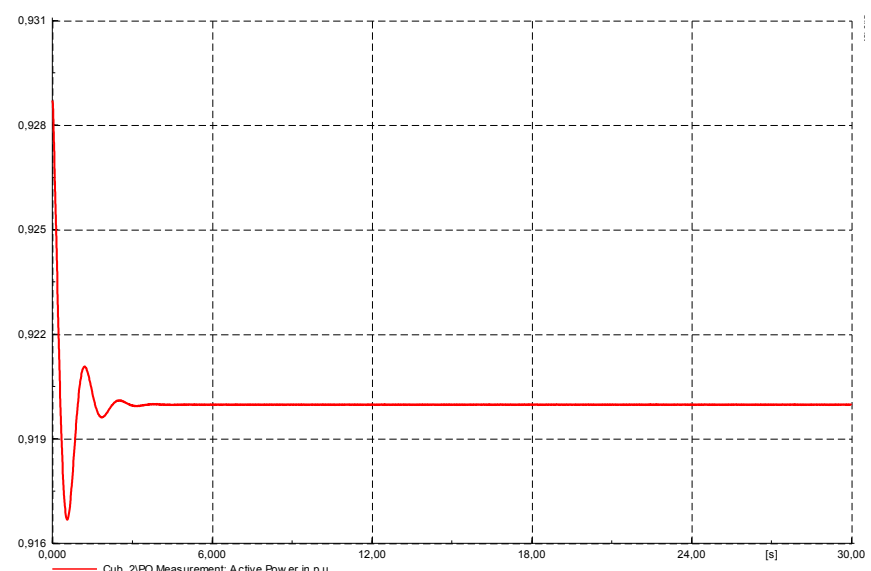

Figure 10. Active Power during normal condition for Type 1B WTG

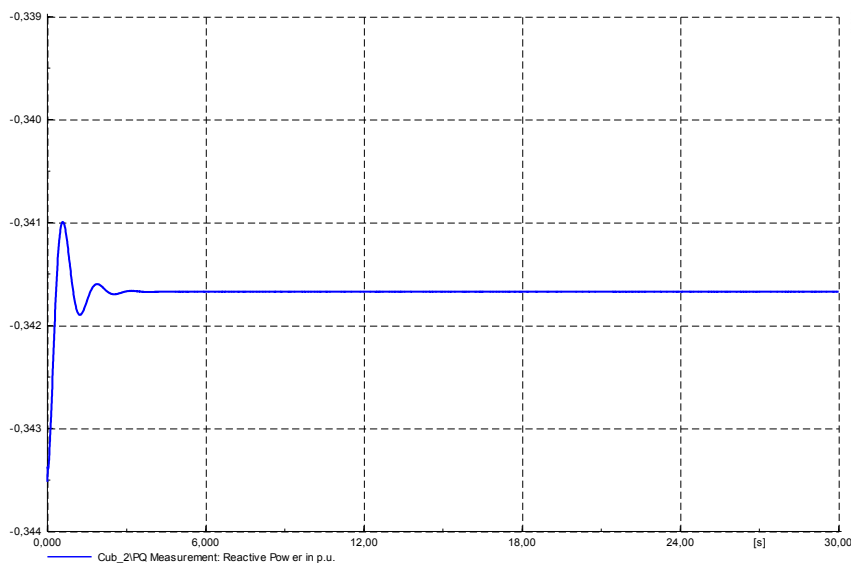

Figure 11. Reactive Power during normal condition for Type 1B WTG

As it can be observe for Type 1 in Figures 8 and 9, the waveforms show the active and reactive power variations following the changes made to the aerodynamic torque.

Otherwise, for both types, a decreasing of the active power production causes the decreasing of the reactive power consumption, while an increasing of the active power production causes an increasing in the reactive power consumption (the negative values reached are higher in the second case). For limiting the absorption of reactive power from the grid, the WTGs are often equipped with capacitor banks.

\subsection{Fault condition}

Because of the balanced RMS simulation method is used, only symmetrical faults are allowed.

Hence, a three-phase short circuit is defined at $4 \mathrm{~s}$ in the LV Terminal for Types $1 \mathrm{~A}$ and $1 \mathrm{~B}$, and it is cleared at $4.2 \mathrm{~s}$ (the simulation time is now $10 \mathrm{~s}$ for Type $1 \mathrm{~A}$ and $30 \mathrm{~s}$ for Type 1B). During the fault, the voltage reaches a minimum value of 0.3933 p.u., as it is represented in Figure 12.

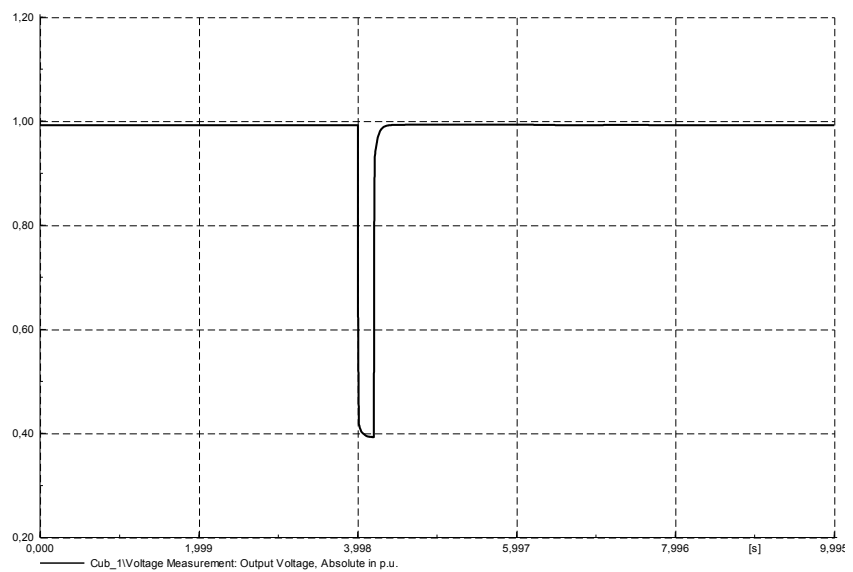

Figure 12. Voltage measurement during fault condition

Figures 13-16 show the waveforms that represent the behaviour of the active and reactive power for both types during the fault condition. 


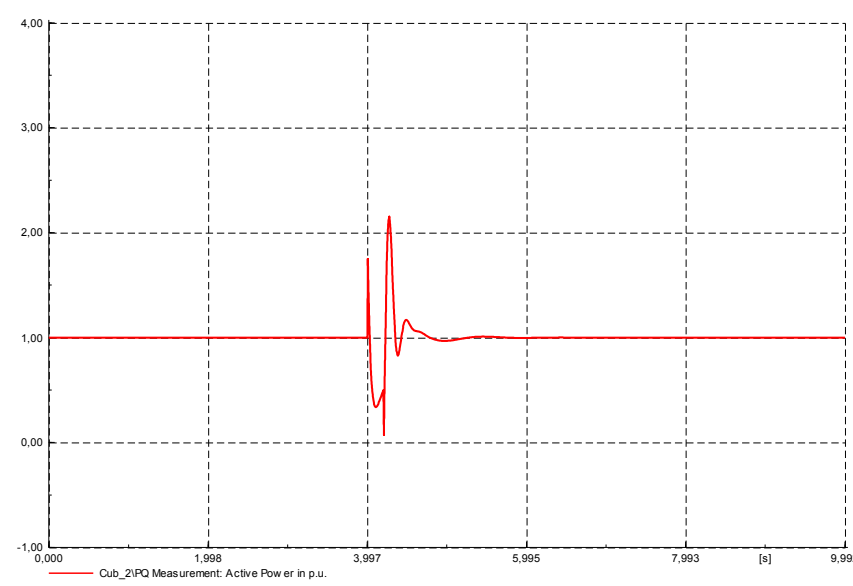

Figure 13. Active Power during fault condition for Type 1A

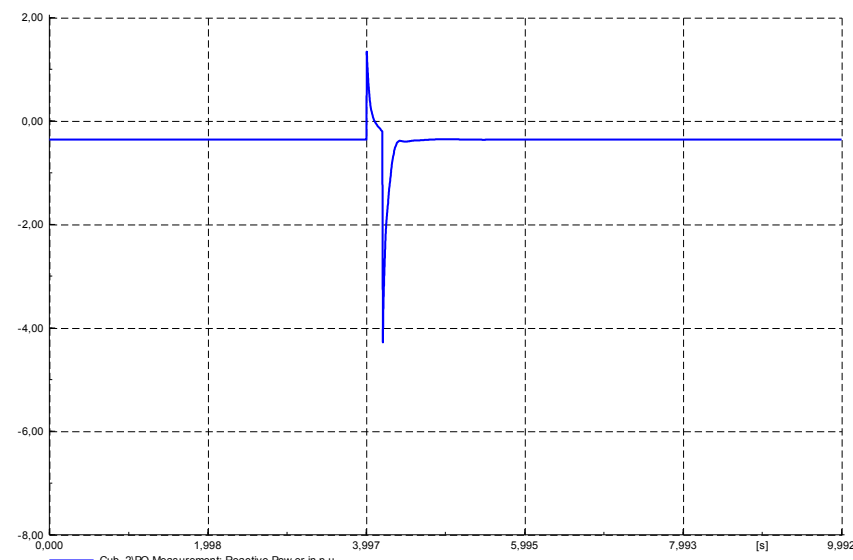

Figure 14. Reactive Power during fault condition for Type 1A

For Type 1A, there is a decreasing of the active power injection during the fault. At $4.2 \mathrm{~s}$, once the fault has been cleared, there is a peak that represents the sudden injection of active power, and this situation back to normal after a certain period of time.

Coinciding with the active power's peak, the WTG presents a deep consumption of reactive power, reaching a value of $-5.6 \mathrm{p} . \mathrm{u}$.

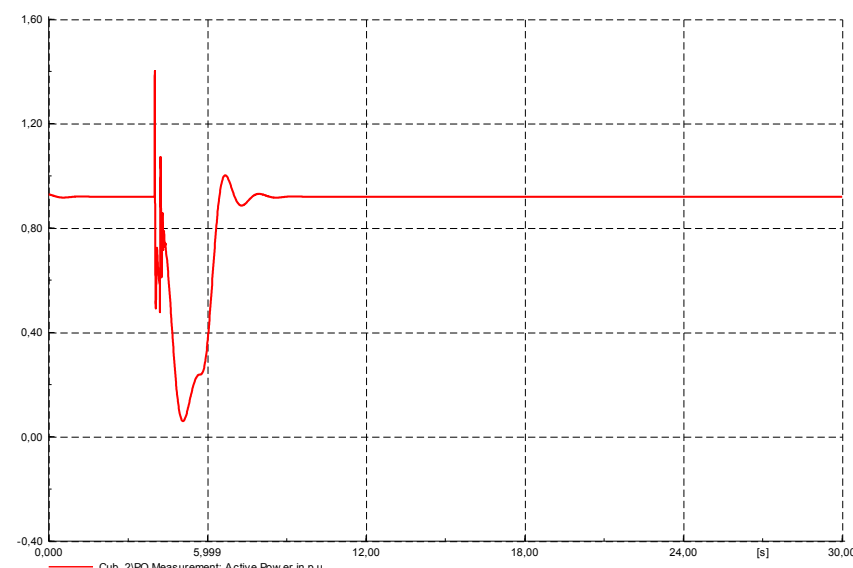

Figure 15. Active Power during fault condition for Type 1B

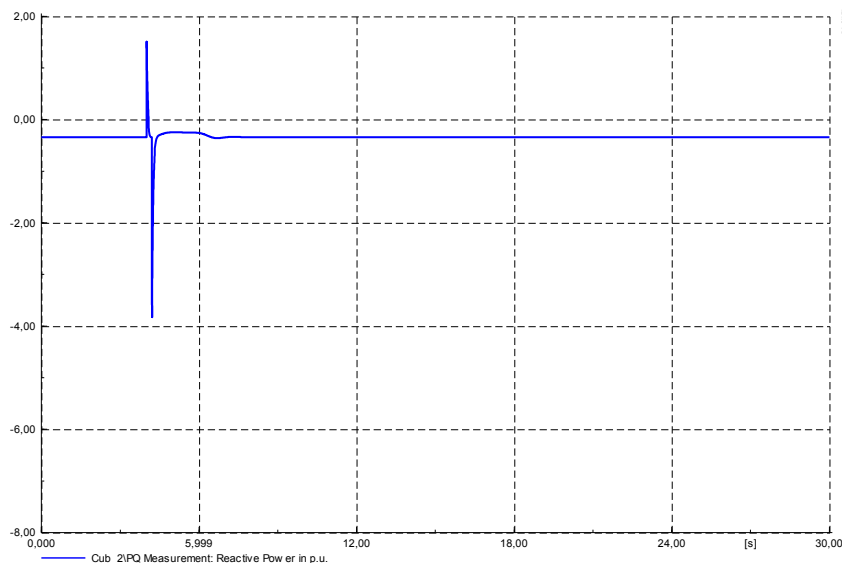

Figure 16. Reactive Power during fault condition for Type 1B

In Type 1B, the main purpose of the Blade Angle Control model is trying to control the variation that leads to the abrupt injection of active power once the fault is cleared.

Thus, as it can be seen in Figure 15, the control model tries to maintain the active power injection in low values during a certain period of time, also avoiding having such a sudden peak of injection like the one in Type 1A. The mentioned value of time is consulted in the lookup table within the control model, and it will depend on the minimum value of voltage reached during the fault. Regarding the current case, the lookup table time has a value of approximately $1.7 \mathrm{~s}$, which means that the active power will remain at a low value during the time of duration of the fault plus the lookup table time. After that, the fault will have being cleared, and the active power will return to its normal value without great oscillations.

\section{Conclusion}

It can be said that these IEC models implemented in PF represent faithfully the real behaviour of actual WTG (both during fault conditions and normal conditions). The appropriate changes made in the blocks as well as the simulation results corroborate these facts. Otherwise, the graphical representation of electrical systems in PF is a very useful and intuitive tool, especially when having them with a complicated structure of multi-input and multi-output signals. Blocks diagrams and frame definitions are highly suitable ways of constructing and relating linear and non-linear DSL models with user predefined or built-in models (i.e. generators), as it is the case of the WTG's analysis.

\section{Acknowledgement}

The authors would like to thank the "Ministerio de Economía y Competitividad" and European Union FEDER, which supported this work under project ENE2016-78214-C2-1-R. 


\section{References}

[1] Honrubia-Escribano, A.; Martín-Martínez, S.; Estanqueiro, A.; Jiménez Buendía, F.; Gómez Lázaro, E. Simplified wind turbine models for wind energy integration into power systems. In Proceedings of the European Wind Energy Conference, Paris, France, 17-20 November 2015; p. 6.

[2] Honrubia Escribano, A.; Gómez-Lázaro, E.; ViguerasRodríguez, A.; Molina-García, A.; Fuentes, J.A.; Muljadi, E. Assessment of DFIG simplified model parameters using field test data. In Proceedings of the IEEE Power Electronics and Machines in Wind Applications (PEMWA), Denver, CO, USA, 16-18 July 2012; pp. 1-7.

[3] Honrubia-Escribano, A.; Jiménez-Buendía, F.; MolinaGarcía, A.; Fuentes-Moreno, J.; Muljadi, E.; Gómez-Lázaro, E. Analysis of Wind Turbine Simulation Models: Assessment of Simplified versus Complete Methodologies. In Proceedings of the XVII International Symposium on Electromagnetic Fields in Mechatronics, Electrical and Electronic Engineering, Valencia, Spain, 10-12 September 2015; p. 8.

[4] A. J. Pujante, J. A. Fuentes, E. Gómez-Lázaro, A. V. Rodriguez, and A. Molina-García, "Performance comparison of a 2 MW DFIG wind turbine model under wind speed variations," in European Wind Energy Conference and Exhibition, 2009, pp. 121-124.

[5] IEC 61400-27-1, "Wind Turbines. Electrical simulation models-Generic models", February 2015.

[6] Haoran Zhao, "Coordinated control of wind power and energy storage”, CEE-PhD-2014, Kongens Lyngby, 2014.

[7] Anca D. Hansen, "Dynamic wind turbine models in power system simulation tool DIgSILENT", Riso-R-1400 (ed.2)(EN), August 2007.

[8] Anca D. Hansen, Poul Sorensen, Florin Iov, Frede Blaabjerg, "Initialisation of Grid-Connected Wind Turbine Models in Power-System Simulations", Wind Energy Volume 27, No. 1, 2003, pp. 21-38.

[9] M. Vinicio Ullauri Rosillo, "Estructuración de una base de datos del S.N.I. ecuatoriano en DIgSILENT PowerFactory para análisis de estabilidad con modelos validados de sus unidades de generación”, Quito, Mayo 2015.

[10] Francisco M. González-Longatt, "PowerFactory Applications for Power System Analysis", Springer International Publishing Switzerland, 2014.

[11] DIgSILENT PowerFactory, "Application Guide - Dynamic Modelling Tutorial, DigSILENT Technical Documentation”, July 2014.

[12] DIgSILENT GmbH, "PowerFactory User's Manual Version 15, Online Edition”, 2013. 